\title{
Role of the Glucosamine Pathway in Fat-induced Insulin Resistance
}

\author{
Meredith Hawkins, Nir Barzilai, Rong Liu, Meizhu Hu, Wei Chen, and Luciano Rossetti \\ Division of Endocrinology and Diabetes Research and Training Center, Albert Einstein College of Medicine, New York 10461
}

\begin{abstract}
To examine whether the hexosamine biosynthetic pathway might play a role in fat-induced insulin resistance, we monitored the effects of prolonged elevations in FFA availability both on skeletal muscle levels of UDP- $N$-acetyl-hexosamines and on peripheral glucose disposal during 7-h euglycemichyperinsulinemic $(\sim 500 \mu \mathrm{U} / \mathrm{ml})$ clamp studies. When the insulin-induced decrease in the plasma FFA levels (to $\sim 0.3$ $\mathrm{mM}$ ) was prevented by infusion of a lipid emulsion in 15 conscious rats (plasma FFA $\sim 1.4 \mathrm{mM}$ ), glucose uptake $(5-7 \mathrm{~h}=32.5 \pm 1.7 \mathrm{vs} 0-2 \mathrm{~h}=45.2 \pm 2.8 \mathrm{mg} / \mathrm{kg}$ per $\mathrm{min} ; P<$ $0.01)$ and glycogen synthesis $(P<0.01)$ were markedly decreased. During lipid infusion, muscle UDP- $N$-acetyl-glucosamine (UDP-GlcNAc) increased by twofold (to 53.4 \pm 1.1 at $3 \mathrm{~h}$ and to $55.5 \pm 1.1 \mathrm{nmol} / \mathrm{gram}$ at $7 \mathrm{~h}$ vs $20.4 \pm 1.7$ at $0 \mathrm{~h}$, $P<0.01$ ) while glucose-6-phosphate (Glc-6-P) levels were increased at $3 \mathrm{~h}(475 \pm 49 \mathrm{nmol} / \mathrm{gram})$ and decreased at $7 \mathrm{~h}$ (133 \pm 7 vs $337 \pm 28 \mathrm{nmol} / \mathrm{gram}$ at $0 \mathrm{~h}, P<0.01)$.

To discern whether such an increase in the skeletal muscle UDP-GIcNAc concentration could account for the development of insulin resistance, we generated similar increases in muscle UDP-GIcNAc using three alternate experimental approaches. Euglycemic clamps were performed after prolonged hyperglycemia (18 $\mathrm{mM}, n=10)$, or increased availability of either glucosamine $(3 \mu \mathrm{mol} / \mathrm{kg}$ per min; $n=10)$ or uridine $(30 \mu \mathrm{mol} / \mathrm{kg}$ per min; $n=4)$. These conditions all resulted in very similar increases in the skeletal muscle UDP-GlcNAc (to $\sim 55 \mathrm{nmol} / \mathrm{gram}$ ) and markedly impaired glucose uptake and glycogen synthesis.

Thus, fat-induced insulin resistance is associated with: $(a)$ decreased skeletal muscle Glc-6-P levels indicating defective transport/phosphorylation of glucose; (b) marked accumulation of the endproducts of the hexosamine biosynthetic pathway preceding the onset of insulin resistance. Most important, the same degree of insulin resistance can be reproduced in the absence of increased FFA availability by a similar increase in skeletal muscle UDP- $N$-acetyl-hexosamines. In conclusion, our results support the hypothesis that increased FFA availability induces skeletal muscle insulin re-
\end{abstract}

\footnotetext{
This work was presented in preliminary form at the 56th annual meeting of the American Diabetes Association, 8-11 June 1996, in San Francisco, CA.

Address correspondence to Luciano Rossetti, Division of Endocrinology, Department of Medicine, Albert Einstein College of Medicine, 1300 Morris Park Avenue, Bronx, NY 10461. Phone: 718-4304118/4215; FAX: 718-430-8557; E-mail: rossetti@aecom.yu.edu

Received for publication 6 December 1996 and accepted in revised form 13 February 1997.
}

J. Clin. Invest.

(C) The American Society for Clinical Investigation, Inc. 0021-9738/97/05/2173/10 \$2.00

Volume 99, Number 9, May 1997, 2173-2182 sistance by increasing the flux of fructose-6-phosphate into the hexosamine pathway. (J. Clin. Invest. 1997. 99:21732182.) Key words: insulin resistance - glucosamine - free fatty acids $\bullet$ UDP- $N$-acetyl-glucosamine • glucose uptake

\section{Introduction}

Non-insulin-dependent diabetes mellitus (NIDDM) ${ }^{1}$ is associated with a marked impairment in the ability of insulin to stimulate glucose uptake in skeletal muscle (1) and most patients with NIDDM are obese and present with both hyperlipidemia and hyperglycemia (2). Inadequate suppression of fatty acid oxidation by insulin is a common feature of various forms of insulin resistance in humans $(2,3)$ and a strong, inverse correlation between glucose and lipid oxidation has been observed in obese subjects with NIDDM (4). Randle and coworkers first recognized this relationship between carbohydrate and lipid metabolism in heart and diaphragm muscle (5). The ensuing hypothesis was that a glucose-fatty acid cycle operates by decreasing the rate of oxidation of the alternate substrate in the presence of increased availability of the other (6). Recent studies have emphasized that, while an increase in circulating FFA during insulin clamp studies promptly decreased the rate of carbohydrate oxidation, defective glucose uptake could only be detected after 3-4 h of lipid infusion (7-10). The reason for such a delay and the mechanism by which the alterations in substrate oxidation bring about a decrease in glucose uptake are still unknown.

In skeletal muscle, after the transport and phosphorylation of glucose to glucose-6-phosphate (Glc-6-P), the latter is primarily utilized in two major pathways, i.e., glycogen synthesis and glycolysis $(11,12)$. However, $\sim 1-3 \%$ of the incoming glucose which is converted to fructose-6-phosphate (Fru-6-P) enters the hexosamine biosynthetic pathway (Fig. 1), whose major endproducts, UDP- $N$-acetyl-glucosamine (UDP-GlcNAc) and UDP- $N$-acetyl-galactosamine (UDP-GalNAc), serve as substrates in the synthesis of glycoproteins $(13,14)$. Several recent studies have demonstrated that increased flux of Fru-6-P into the hexosamine biosynthetic pathway antagonizes the ability of insulin to stimulate glucose uptake in vivo (15-17) and in isolated cell systems (18-20). The latter represents a likely mechanism by which chronic hyperglycemia results in defective stimulation of glucose transport by insulin $(15,20,21)$. Importantly, the accumulation of UDP- $N$-acetyl-hexosamines in skeletal muscle, an index of the flux through the hexosamine pathway, was highly correlated with the degree of insulin resistance (22-24).

In the presence of increased availability of FFA, the increased concentration of acetyl-CoA, derived in the mitochon-

1. Abbreviations used in this paper: Fru-6-P, fructose-6-phosphate; Gal, galactose; GalNAc, $N$-acetyl-galactosamine; Glc, glucose; GlcN, glucosamine; GlcNAc, $N$-acetyl-glucosamine; Glc-6-P, glucose-6-phosphate; NIDDM, non-insulin-dependent diabetes mellitus; Rd, glucose uptake. 


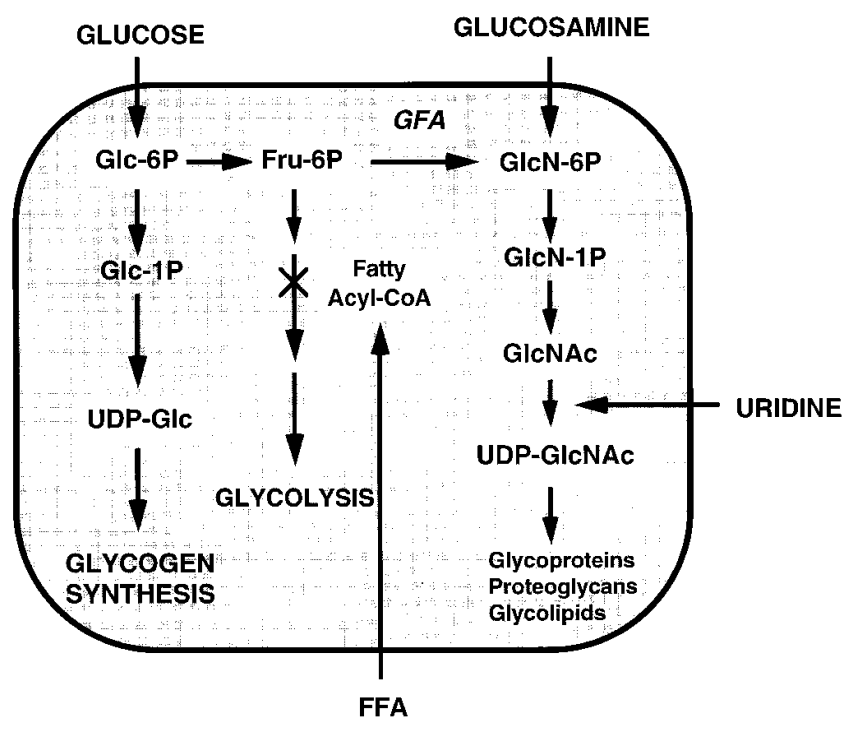

Figure 1. Sites of entry of glucose, GlcN, and uridine into the hexosamine biosynthetic pathway in skeletal muscle and potential role of FFA. After its entry into skeletal muscle cells via the glucose transport system and its rapid phosphorylation to Glc-6-P, glucose is primarily utilized by the pathways of glycogen synthesis and glycolysis. The hexosamine pathway receives $\sim 1-3 \%$ of incoming glucose via the conversion of Fru-6-P to glucosamine-6-phosphate by the ratelimiting enzyme glutamine: fructose-6-phosphate amidotransferase $(G F A)$. Alternatively, infused GlcN enters skeletal muscle cells directly via the glucose transport system and is phosphorylated to GlcN-6-P. Further metabolites are formed by subsequent acetylation and uridylation of GlcN-6-P. Hence infused uridine, which contributes to the intracellular UDP/UTP pool, enhances the formation of downstream products of the hexosamine biosynthetic pathway. The principal endproduct of this pathway, UDP-GlcNAc, serves as substrate in glycoprotein biosynthesis and its accumulation provides an index of the amount of carbon flux through the pathway. Increased FFA availability generates increased acetylCoA which inhibits pyruvate dehydrogenase and thus ultimately the rate of glycolysis. This results in increased accumulation of Fru-6-P and hence increased substrate for the GFA enzyme.

dria from the oxidation of FFA, decreases the rate of pyruvate oxidation via inhibition of pyruvate dehydrogenase (PDH) (25). Furthermore, the entry of Fru-6-P in the glycolytic pathway is also limited by the inhibition of phosphofructokinase (PFK) (Fig. 1) mediated by increased citrate (25) and/or decreased xylulose-5-P concentration (26). This, in turn, is expected to increase the availability of Fru-6-P for the formation of glucosamine-6-P.

Thus, we wish to test the hypothesis that an expanded fructose-6-phosphate pool, whether due to increased phosphorylation of glucose (e.g., hyperglycemia) or to decreased utilization of Fru-6-P via glycolysis (e.g., increased availability of free fatty acids), would increase carbon flux into the GlcN pathway and via increased concentration of its endproducts, UDP- $N$ acetyl-hexosamines, cause an impairment in glucose transport/ phosphorylation. Thus, we examined whether prolonged exposure to high concentrations of free fatty acids would increase flux into the hexosamine biosynthetic pathway, and if so, whether this increase would precede and be sufficient for the development of peripheral insulin resistance.

\section{Methods}

\section{Animals}

45 male Sprague-Dawley rats (Charles River Breeding Laboratories, Inc., Wilmington, MA) were housed in individual cages and subjected to a standard light (6 a.m. to 6 p.m.)-dark (6 p.m. to 6 a.m.) cycle. The rats were anesthetized with intraperitoneal injection of pentobarbital $(50 \mathrm{mg} / \mathrm{kg}$ body weight) and indwelling catheters were inserted into the right internal jugular vein and the left carotid artery, as previously described $(15,27-29)$. The venous catheter was extended to the level of the right atrium and the arterial catheter was advanced to the level of the aortic arch. The in vivo studies were performed 5-7 d after catheter placement.

\section{In vivo clamp studies}

All studies were performed in awake, unstressed rats fasted for $6 \mathrm{~h}$. Euglycemic-hyperinsulinemic (18 $\mathrm{mU} / \mathrm{kg}$ per min) clamp studies were performed in combination with $\left[3-{ }^{3} \mathrm{H}\right]$ glucose infusion as previously described $(15,22,23,27-29)$. A primed-continuous infusion of HPLC purified [ $\left.{ }^{3} \mathrm{H}-3\right]$ glucose (New England Nuclear, Boston, MA; $8-\mu \mathrm{Ci}$ bolus, $0.4 \mu \mathrm{Ci} / \mathrm{min}$ ) was infused throughout the insulin clamp periods to measure the rates of peripheral glucose uptake and glycolysis. A variable infusion of $25 \%$ glucose solution was started at time zero and adjusted every 5-10 min, maintaining basal plasma glucose concentrations $(\sim 7 \mathrm{mM})$ during the insulin clamp studies.

Study 1: High FFA (Fig. 2). To assess the effects of a sustained increase in the availability of free fatty acids on insulin action, continuous infusions of lipid emulsion (Liposyn 10\%; Abbott Laboratories, North Chicago, IL; in 1:2 suspension with heparinized saline) were maintained for 3 (High FFA-3h, $n=5$ ) or $7 \mathrm{~h}$ (High FFA-7h, $n=5$ ) at a rate of $1.5 \mathrm{ml} / \mathrm{h}$ during prolonged euglycemic-hyperinsulinemic (18 mU/kg per min) clamp studies (Fig. 2). To determine whether the continuing presence of high circulating FFA is required for the persistence of insulin resistance once established, lipid infusion was termi-

\section{LIPOSYN 10\% (1.5 ml/h) :}
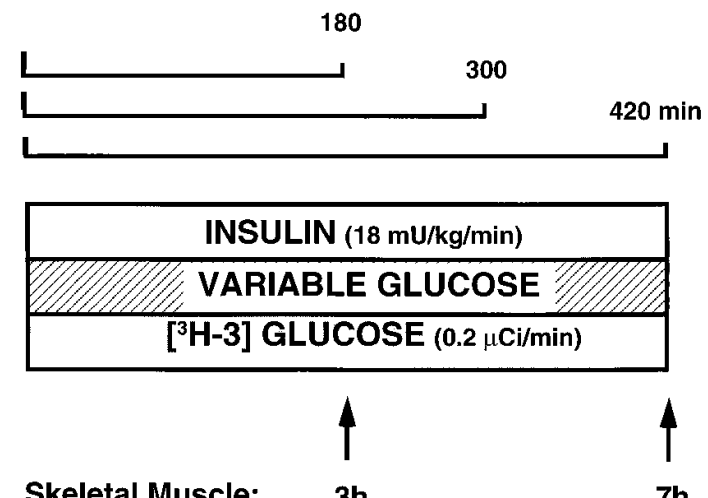

Skeletal Muscle: $\quad 3 h \quad$ Th

Figure 2. Schematic representation of the experimental protocol used to assess the effects of increased FFA availability on peripheral insulin action. Euglycemic, hyperinsulinemic $(18 \mathrm{mU} / \mathrm{kg}$ per min; $\sim 500 \mu \mathrm{U} / \mathrm{ml}$ ) clamps were performed during infusions of lipid emulsion (Liposyn 10\%) in conscious nondiabetic rats $(n=15)$. One subgroup of rats $(n=5)$ was killed, and skeletal muscle samples were obtained, after 3-h hyperinsulinemic clamps with continuous lipid infusion. In another subgroup $(n=5)$ the lipid infusion was terminated at $5 \mathrm{~h}$ while the hyperinsulinemic clamps were maintained for $7 \mathrm{~h}$. In the remaining $n=5$ rats, both the infusion of lipid and the hyperinsulinemic clamps continued for $7 \mathrm{~h}$. Baseline blood glucose levels were maintained throughout the studies using variable infusion rates of a $25 \%$ glucose solution. Tritiated glucose was infused throughout all studies to measure peripheral glucose uptake. 
nated at $5 \mathrm{~h}$ in an additional $n=5$ rats (High FFA-5h) while the hyperinsulinemic clamps were maintained for $7 \mathrm{~h}$.

Three alternate experimental protocols were designed to generate an increase in the flux into the glucosamine pathway in skeletal muscle similar to that measured during lipid infusion in study 1 . The goal of these studies was to determine whether a moderate increase in the skeletal muscle concentration of UDP-GlcNAc would be sufficient to cause impaired insulin action on skeletal muscle glucose uptake.

Study 2: Hyperglycemia. Prolonged hyperglycemia in the presence of basal insulin increases the flux of Fru-6-P into the hexosamine biosynthetic pathway and causes a moderate increase in the skeletal muscle concentrations of UDP-GlcNAc (unpublished observations). In this protocol, euglycemic-hyperinsulinemic $(18 \mathrm{mU} / \mathrm{kg}$ per min) clamp studies $(n=4)$ were used to measure insulin action on glucose fluxes during the initial ( $0-2 \mathrm{~h}$ ) and final $(5-7 \mathrm{~h}) 2 \mathrm{~h}$, while hyperglycemia $(18 \mathrm{mM})$ and basal insulinemia were maintained during the intervening three hours $(2-5 \mathrm{~h})$ using primed-continuous infusions of somatostatin (SRIF, $1.2 \mu \mathrm{g} / \mathrm{kg}$ per min) and insulin replacement $(0.7$ $\mathrm{mU} / \mathrm{kg}$ per min). To verify that prolonged hyperglycemia was able to increase the skeletal muscle concentration of the endproducts of the GlcN pathway, additional rats were killed during the hyperglycemic period at $3(n=3)$ or $5 \mathrm{~h}(n=3)$.

Study 3: High GlcN. An alternative means to reproduce the moderate increase in muscle UDP-GlcNAc measured during the lipid infusion protocol is to infuse GlcN, which directly enters the hexosamine pathway via phosphorylation to GlcN-6-P (15). Thus, euglycemichyperinsulinemic ( $18 \mathrm{mU} / \mathrm{kg}$ per $\mathrm{min}$ ) clamp studies were maintained during the initial and final $2 \mathrm{~h}$ of continuous, 7-h infusions of GlcN ( $3 \mu \mathrm{mol} / \mathrm{kg}$ per min, $n=10$ ). This rate of GlcN infusion is 10 times lower than that used in our previous reports $(15,22,23)$ and was selected to induce an approximate twofold increase in skeletal muscle UDP-GlcNAc levels.

Study 4: High uridine. Recently we have demonstrated that the provision of uridine during insulin clamp studies can increase the efficiency of the uridylation of GlcNAc to UDP-GlcNAc and therefore results in increased accumulation of this endproduct (23). Thus, euglycemic hyperinsulinemic ( $18 \mathrm{mU} / \mathrm{kg}$ per $\mathrm{min}$ ) clamp studies were maintained during the initial and final $2 \mathrm{~h}$ of continuous, 7-h infusions of uridine ( $30 \mu \mathrm{mol} / \mathrm{kg}$ per $\min , n=4)$.

Saline time-control euglycemic-hyperinsulinemic $(18 \mathrm{mU} / \mathrm{kg}$ per min) clamp studies $(7 \mathrm{~h})$ were performed in an additional $(n=6)$ ageand weight-matched nondiabetic rats.

Plasma samples for determination of ${ }^{3} \mathrm{H}$-glucose specific activity (SA) were obtained at 10-min intervals throughout the insulin infusions. Samples for measurement of plasma insulin, FFA and GlcN concentrations were obtained at times $0,60,120,180,240,300,360$, and $420 \mathrm{~min}$. The total volume of blood sampled was $\sim 5.0 \mathrm{ml} / \mathrm{study}$; to prevent volume depletion and anemia, a solution $(1: 1 \mathrm{vol} / \mathrm{vol})$ of $\sim 6.0$ $\mathrm{ml}$ of fresh blood (obtained by heart puncture from a littermate of the test animal) and heparinized saline $(10 \mathrm{U} / \mathrm{ml})$ was infused. At the end of the in vivo studies, rats were anesthetized (pentobarbital $60 \mathrm{mg} / \mathrm{kg}$ body weight, i.v.), the abdomen was quickly opened, and rectus abdominal muscle was freeze-clamped in situ with aluminum tongs precooled in liquid nitrogen (12-15). The time from injection of the anesthetic to freeze clamping of the muscle was $<30 \mathrm{~s}$. All tissue samples were stored at $-80^{\circ} \mathrm{C}$ for subsequent analysis. The study protocol was reviewed and approved by the Institutional Animal Care and Use Committees of the Albert Einstein College of Medicine.

\section{Whole body glycolysis}

The rates of glycolysis were estimated as previously described (27-29). Briefly, plasma-tritiated water specific activity was determined by liquid scintillation counting of the protein-free supernatant (Somogyi filtrate) before and after evaporation to dryness. Since tritium on the C-3 position of glucose is lost to water during glycolysis, it can be assumed that plasma tritium is present either in the form of tritiated water or [3$\left.{ }^{3} \mathrm{H}\right]$ glucose (27). The rates of glycogen synthesis were estimated as the difference between the rates of glucose uptake and glycolysis.

\section{Analytical procedures}

Plasma glucose was measured by the glucose oxidase method (Glucose Analyzer II; Beckman Instruments, Inc., Palo Alto, CA) and plasma insulin by radioimmunoassay using rat and porcine insulin standards. Quantitative determination of plasma FFA levels was performed using an acyl-CoA oxidase-based colorimetric kit (Wako, Osaka, Japan). Plasma ${ }^{3} \mathrm{H}$-glucose radioactivity was measured in duplicate on the supernatants of $\mathrm{Ba}(\mathrm{OH})_{2}$ and $\mathrm{ZnSO}_{4}$ precipitates of plasma samples after evaporation to dryness to eliminate tritiated water. Regression analysis of the slopes of ${ }^{3} \mathrm{H}_{2} \mathrm{O} \mathrm{Ra}$ (used in the calculation of the rates of glycolysis) was performed at 60 -min intervals throughout the study. Muscle glucose-6-phosphate concentrations were measured spectrophotometrically as described by Michal (30).

Muscle Uridinediphosphoglucose (UDP-Glc), Uridinediphosphogalactose (UDP-Gal), UDP- $N$-acetyl-glucosamine (UDP-GlcNAc) and UDP- $N$-acetyl-galactosamine (UDP-GalNAc) concentrations were obtained through two sequential chromatographic separations and UV detection $(12,31,32)$. UDP-GlcNAc and UDP-GalNAc coeluete with UDP-Glc and UDP-Gal during the solid phase extraction. The retention time for UDP-Glc, UDP-Gal, UDP-GlcNAc, and UDP-GalNAc were $28.5,30.7,33.9$ and $35.4 \mathrm{~min}$, respectively. Plasma GlcN concentrations were determined by HPLC after quantitative derivitization with phenyl isothiocyanate (PITC) as described by Anumula and Taylor (33). All HPLC analysis were performed on an HPLC system (Waters Associates, Milford, MA) using a reverse-phase, ion pairing isocratic method, on two C18T (Supelco Inc., Bellefonte, PA) reverse-phase columns $(0.46 \times 25 \mathrm{~cm})$ in series.

All values are presented as the mean \pm SE. Comparisons between groups were made using repeated measures analysis of variance where appropriate. Where F-ratios were significant, further comparisons were made using student $t$ tests (paired difference test and smallsample test for independent samples).

\section{Results}

\section{Study 1: High FFA}

General and biochemical characteristics of the experimental animals (Table I, Fig. 3). At the time of the study, the mean body weight of the animals was $309 \pm 16$ grams. The mean plasma glucose, FFA and insulin concentrations at baseline $(0 \mathrm{~h})$ were similar in all groups (Table I; $P>0.1$ ).

Table I. Plasma Concentrations of Glucose, Free Fatty Acids, and Insulin during 7-h Euglycemic-Hyperinsulinemic Clamp Studies with Continuous Infusion of Lipid Emulsion (High FFA) or Saline

\begin{tabular}{lccc}
\hline \multicolumn{1}{c}{ High FFA } & $0 \mathrm{~h}$ & $3 \mathrm{~h}$ & $7 \mathrm{~h}$ \\
\hline & $n=15$ & $n=15$ & $n=5$ \\
Glucose $(\mathrm{mM})$ & $7.4 \pm 0.1$ & $7.5 \pm 0.1$ & $7.5 \pm 0.2$ \\
Free fatty acids $(\mathrm{mM})$ & $0.9 \pm 0.1$ & $1.3 \pm 0.1^{*}$ & $1.5 \pm 0.2^{*}$ \\
Insulin $(\mu \mathrm{U} / \mathrm{ml})$ & $26 \pm 5$ & $537 \pm 94^{\ddagger}$ & $595 \pm 92^{\ddagger}$ \\
\hline \multicolumn{1}{c}{ Saline } & $0 \mathrm{~h}$ & $3 \mathrm{~h}$ & $7 \mathrm{~h}$ \\
\hline & $n=6$ & $n=6$ & $n=6$ \\
& $7.3 \pm 0.2$ & $7.5 \pm 0.1$ & $7.3 \pm 0.1$ \\
Glucose $(\mathrm{mM})$ & $0.9 \pm 0.1$ & $0.3 \pm 0.2^{*}$ & $0.4 \pm 0.1^{*}$ \\
Free fatty acids $(\mathrm{mM})$ & $37 \pm 6$ & $554 \pm 63^{\ddagger}$ & $582 \pm 72^{\ddagger}$ \\
Insulin $(\mu \mathrm{U} / \mathrm{ml})$ & & & \\
& & &
\end{tabular}

$* P<0.05$ vs $0 \mathrm{~h} ;{ }^{\ddagger} P<0.001$ vs $0 \mathrm{~h}$. 

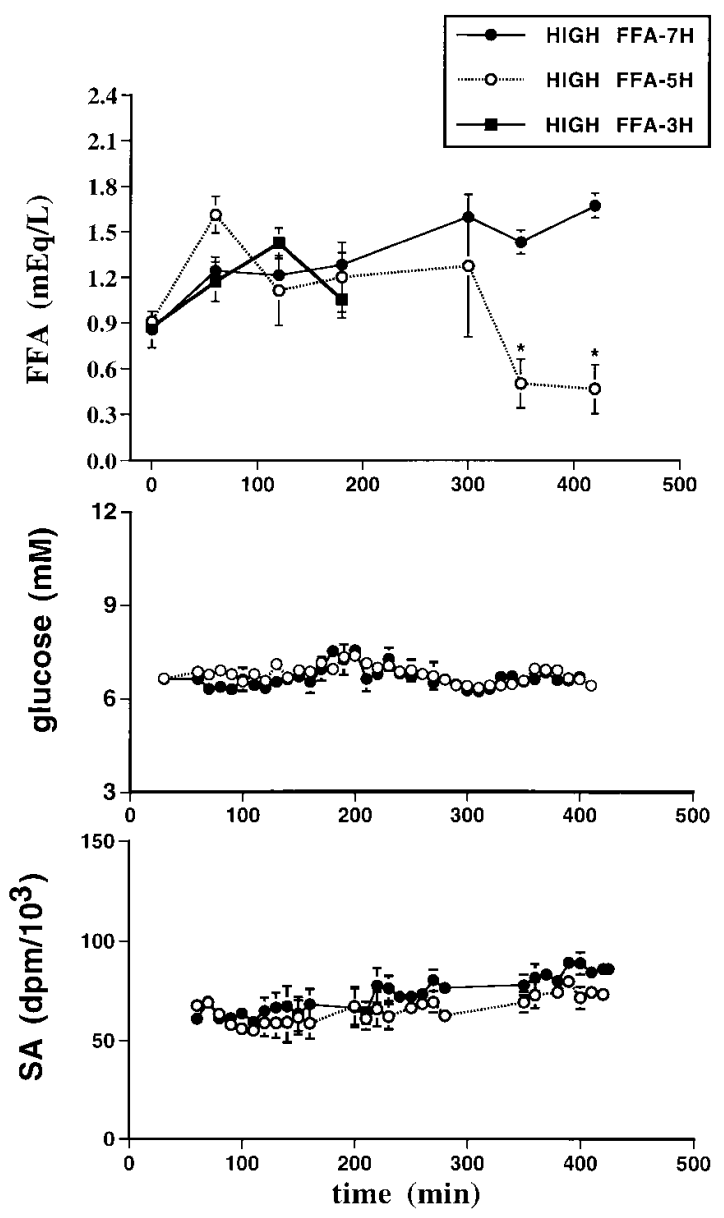

Figure 3. Plasma concentrations of FFA, glucose, and the specific activity of tritiated glucose $(S A)$ during euglycemic-hyperinsulinemic clamp studies with continuous infusion of lipid emulsion for $3 \mathrm{~h}$ (High $F F A-3 H ; n=5), 7 \mathrm{~h}($ High FFA-7H; $n=5)$, or during $7 \mathrm{~h}$ euglycemic-hyperinsulinemic clamp studies with termination of lipid infusion at $5 \mathrm{~h}($ High FFA $-5 H ; n=5) . * P<0.01$ vs High FFA-7H.

In vivo metabolic parameters (Fig. 4). The plasma glucose concentration was maintained at basal levels throughout all clamp studies. The plasma insulin levels were maintained constant at $\sim 500 \mu \mathrm{U} / \mathrm{ml}$ during the 7-h studies. The coefficients of variation in plasma glucose and insulin levels were $<5$ and $10 \%$, respectively, in all studies. Consistent with previous observations (12), during the time-control insulin clamp studies the plasma FFA concentration fell markedly to $\sim 0.3 \mathrm{mM}$ and significant reductions in the rates of glucose infusion $(5-7 \mathrm{~h}=$ $30.5 \pm 3.6$ vs $0-2 \mathrm{~h}=33.3 \pm 3.0 \mathrm{mg} / \mathrm{kg}$ per min; $P>0.05)$ or glucose uptake $(5-7 \mathrm{~h}=38.2 \pm 2.3 \mathrm{vs} 0-2 \mathrm{~h}=43.2 \pm 2.5 \mathrm{mg} / \mathrm{kg}$ per min; $P>0.05$ ) could not be detected. During similar 3- and 7-h insulin clamp studies the plasma FFA levels were maintained $50 \%$ above basal levels $(\sim 1.4 \mathrm{mM})$ by infusion of lipid emulsion (Table I, Fig. 3). In the protocol in which the lipid infusion was terminated at $5 \mathrm{~h}$ (High FFA-5h), plasma FFA levels fell rapidly below baseline levels $(\sim 0.4 \mathrm{mM})$. Lipid infusion resulted in significant and progressive decreases in insulin-mediated glucose disposal which became statistically significant at $\sim 3 \mathrm{~h}$ and reached a maximum by $\sim 5 \mathrm{~h}$. Fig. 4 depicts the rates of peripheral glucose uptake (Rd), glycogen synthesis (GS)
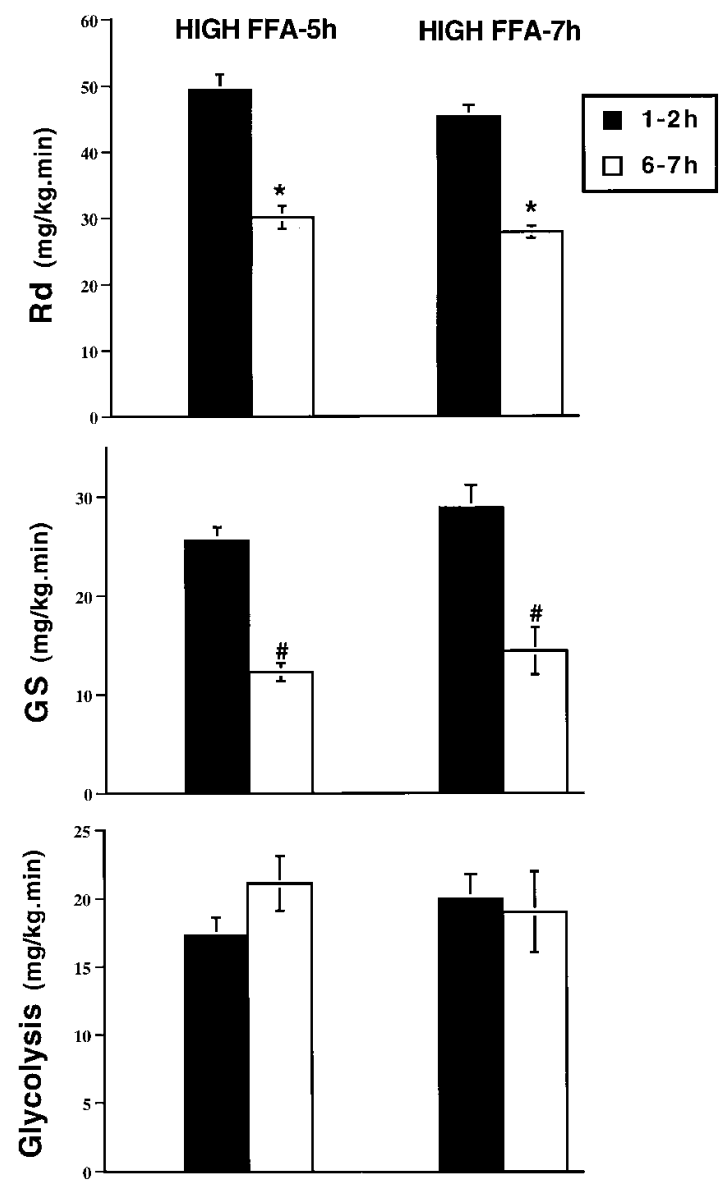

Figure 4. Glucose uptake $(R d)$, glycogen synthesis $(G S)$, and glycolysis plotted at the initial and final $2 \mathrm{~h}$ during 7 -h euglycemic-hyperinsulinemic clamp studies with continuous infusion of lipid emulsion (High FFA-7h; $n=5$ ) or during 7-h euglycemic-hyperinsulinemic clamp studies with termination of lipid infusion at $5 \mathrm{~h}$ (High FFA-5h; $n=5)$. ${ }^{*} P<0.005 ;{ }^{\sharp} P<0.001$ vs $1-2 \mathrm{~h}$.

and glycolysis during the initial (0-2 h) and the final (5-7 h) $2 \mathrm{~h}$ of the High FFA-5h and the High FFA-7h protocols. It is noteworthy that the percent reductions in the rates of peripheral glucose fluxes were similar over the course of $7 \mathrm{~h}$ in both the High FFA-7h $(\Delta \mathrm{Rd}:-29.0 \%)$ and the High FFA-5h $(\Delta \mathrm{Rd}$ : $-29.7 \%$ ) protocols. The observed decreases in $\mathrm{Rd}$ in both groups were largely due to the marked, $\sim 50 \%$ reduction in the rate of glycogen synthesis. Likewise, the time course of these effects was also similar in both groups, with half-maximal reductions in $\mathrm{Rd}$ and GS by $\sim 3.5 \mathrm{~h}$.

Skeletal muscle metabolites (Fig. 5; Table II). Results obtained during the 7-h insulin clamp studies were superimposable in the groups receiving lipid infusion for 5 or $7 \mathrm{~h}$ and are therefore presented together. Skeletal muscle glucose-6-phosphate concentrations were significantly elevated above basal levels after the initial $3 \mathrm{~h}$ of lipid infusion $(P<0.05)$. There were subsequent reductions in Glc-6-P levels after $5 \mathrm{~h}(P<$ 0.01 ; data not shown) and $7 \mathrm{~h}(P<0.01)$ of lipid infusion to levels which were markedly lower than those measured either at baseline (Fig. 5) or at the end of the time control studies $(291 \pm 30 \mathrm{nmol} / \mathrm{grams} ; P<0.01)$. The latter marked decline in 

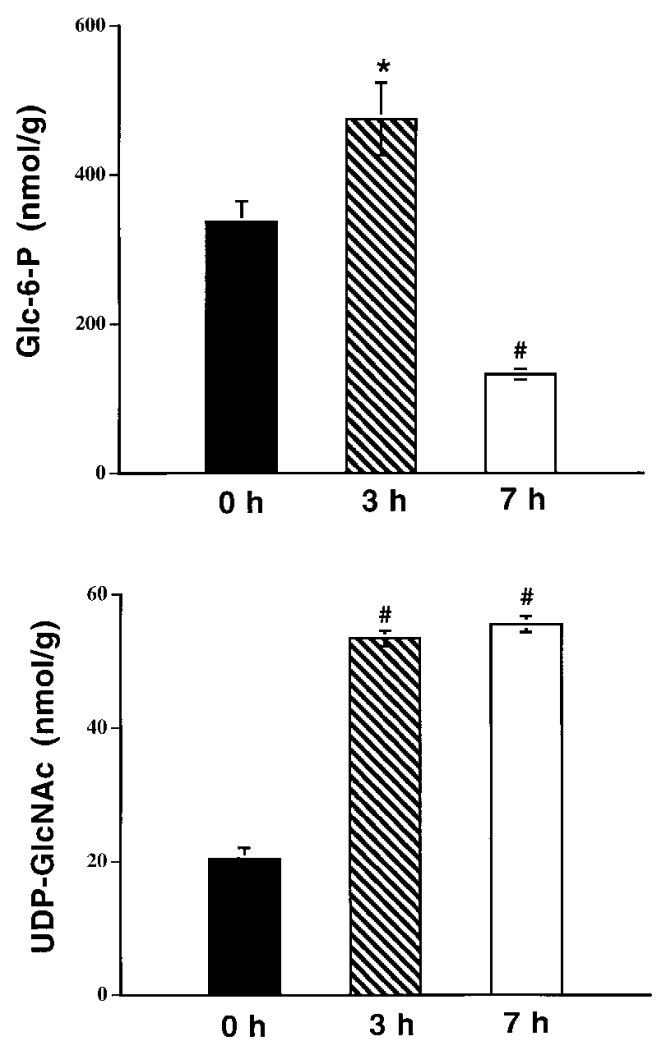

Figure 5. Skeletal muscle concentrations of Glc-6-P and UDPGlcNAc at the end of euglycemic-hyperinsulinemic clamp studies with continuous infusions of lipid emulsion for $3 \mathrm{~h}$ (High FFA-3h; $n=5$ ) or $7 \mathrm{~h}$ (High FFA-7h; $n=5) .0$ h levels $(n=6)$ represent tissue levels after 7-h euglycemic-hyperinsulinemic clamp studies with saline infusion. ${ }^{*} P<0.025$; ${ }^{\#} P<0.01$.

skeletal muscle Glc-6-P concentrations was temporally related with the onset of insulin resistance and likely reflected the development of impaired glucose transport and/or phosphorylation. Pronounced elevations of the skeletal muscle concentrations of the UDP- $N$-acetyl-hexosamines (UDP-GlcNAc and UDP-GalNAc) were already evident by $3 \mathrm{~h}$ and persisted after $7 \mathrm{~h}$ of continuous lipid infusion (Fig. 5). The skeletal muscle concentrations of UDP-Glc and UDP-galactose were similar throughout the FFA infusions (UDP-Glc: High FFA-3h =

Table II. Skeletal Muscle UDP-Glc, UDP-Gal, UDP-GlcNAc, and UDP-GalNAc Concentrations at the End of 7-h Insulin Clamp Studies in 6-h Fasted Rats in the Presence of Increased Free Fatty Acids (High FFA) Relative to 7-h Insulin Clamp Studies in the Presence of Saline Infusion (Saline)

\begin{tabular}{lrr}
\hline & \multicolumn{1}{c}{ Saline } & High FFA \\
\hline UDP-Glc (nmol/gram) & $22.3 \pm 6.1$ & $23.3 \pm 1.0$ \\
UDP-Gal (nmol/gram) & $5.2 \pm 0.5$ & $4.8 \pm 0.3$ \\
UDP-GlcNAc (nmol/gram) & $26.9 \pm 7.3$ & $55.5 \pm 1.1^{\ddagger}$ \\
UDP-GalNAc (nmol/gram) & $9.4 \pm 2.3$ & $15.1 \pm 2.2^{*}$ \\
Ratio UDP-GlcNAc/UDP-Glc & $1.2 \pm 0.5$ & $2.3 \pm 0.1^{*}$
\end{tabular}

${ }^{*} P<0.05 ;{ }^{\ddagger} P<0.01$ vs saline. Values are mean \pm SE.
$22.2 \pm 2.0 \mathrm{nmol} / \mathrm{grams}$, High FFA $-5 \mathrm{~h}=22.3 \pm 2.2 \mathrm{nmol} / \mathrm{grams}$ and High FFA-7h $=23.3 \pm 1.0 \mathrm{nmol} / \mathrm{gram})$, and comparable with those in saline-infused controls. However, the ratios of the skeletal muscle concentrations of UDP-GlcNAc to UDPGlc were significantly increased with lipid infusion over all time courses. Animals killed at $7 \mathrm{~h}$ with termination of lipid infusion at $5 \mathrm{~h}$ (High FFA-5h) also exhibited skeletal muscle concentrations of UDP-GlcNAc $(42.2 \pm 1.0 \mathrm{nmol} / \mathrm{gram} ; P<$ 0.01 ) that were persistently elevated above the basal levels and above the levels measured at the completion of the time control studies $(26.9 \pm 3.2 \mathrm{nmol} / \mathrm{grams})$.

Thus, the persistence of fat-induced insulin resistance once established did not depend upon the continuous presence of elevated circulating FFA. Furthermore, the FFA-induced impairment in the actions of insulin on glucose uptake and glycogen synthesis was associated with an early increase in the skeletal muscle concentration of hexose-phosphates and with the accumulation of endproducts of the GlcN pathway. To investigate whether increased flux into the GlcN pathway could, in fact, be responsible for the effects of lipid on insulin action, additional studies were designed to predictably increase the flux into the GlcN pathway in the absence of prolonged elevations in circulating FFA.

Study 2 (hyperglycemia), Study 3 (High GlcN), and Study 4 (High uridine)

General and biochemical characteristics of the experimental animals (Table III). At the time of the study, the mean body weights of the animals in all groups were similar: $299 \pm 12$ grams with hyperglycemia, 276 \pm 15 grams with GlcN infusion and $251 \pm 15$ grams with uridine infusion. The mean plasma glucose, FFA, GlcN, and insulin concentrations at baseline $(0 \mathrm{~h})$ were similar in all groups (Table III; $P>0.1$ ).

Table III. Plasma Concentrations of Glucose, Free Fatty Acids, Glucosamine, and Insulin during 7-h Hyperglycemic Clamp Studies with Low, Physiologic Insulin (Hyperglycemia), and 7-h Euglycemic-Hyperinsulinemic Clamp Studies with Infusions of Glucosamine (High GlcN) and Uridine (High Uridine)

\begin{tabular}{lccc}
\hline & Hyperglycemia & High GlcN & High uridine \\
\hline Glucose $(\mathrm{mM})$ & & & \\
$0 \mathrm{~h}$ & $7.4 \pm 0.2$ & $7.2 \pm 0.1$ & $7.3 \pm 0.2$ \\
$1-2 \mathrm{~h}$ & $7.5 \pm 0.3$ & $7.3 \pm 0.1$ & $7.4 \pm 0.3$ \\
$6-7 \mathrm{~h}$ & $7.4 \pm 0.2$ & $7.3 \pm 0.2$ & $7.2 \pm 0.3$ \\
Free fatty acids (mM) & & & \\
$0 \mathrm{~h}$ & $1.0 \pm 0.1$ & $0.9 \pm 0.1$ & $0.8 \pm 0.3$ \\
$1-2 \mathrm{~h}$ & $0.3 \pm 0.1^{*}$ & $0.4 \pm 0.1^{*}$ & $0.4 \pm 0.2^{*}$ \\
$6-7 \mathrm{~h}$ & $0.4 \pm 0.2^{*}$ & $0.3 \pm 0.1^{*}$ & $0.3 \pm 0.2^{*}$ \\
Glucosamine (mM) & & & \\
$0 \mathrm{~h}$ & $<0.02$ & $<0.02$ & $<0.02$ \\
$1-2 \mathrm{~h}$ & $<0.02$ & $0.13 \pm 0.03^{*}$ & $<0.02$ \\
$6-7 \mathrm{~h}$ & $<0.02$ & $0.16 \pm 0.04^{*}$ & $<0.02$ \\
Insulin $(\mu \mathrm{U} / \mathrm{ml})$ & & & \\
$0 \mathrm{~h}$ & $34 \pm 6$ & $37 \pm 9$ & $28 \pm 6$ \\
$1-2 \mathrm{~h}$ & $548 \pm 6^{*}$ & $608 \pm 72^{*}$ & $566 \pm 91^{*}$ \\
$6-7 \mathrm{~h}$ & $536 \pm 5^{*}$ & $533 \pm 78^{*}$ & $618 \pm 89^{*}$ \\
& & & \\
\hline
\end{tabular}

$* P<0.01$ vs $0 \mathrm{~h}$. 


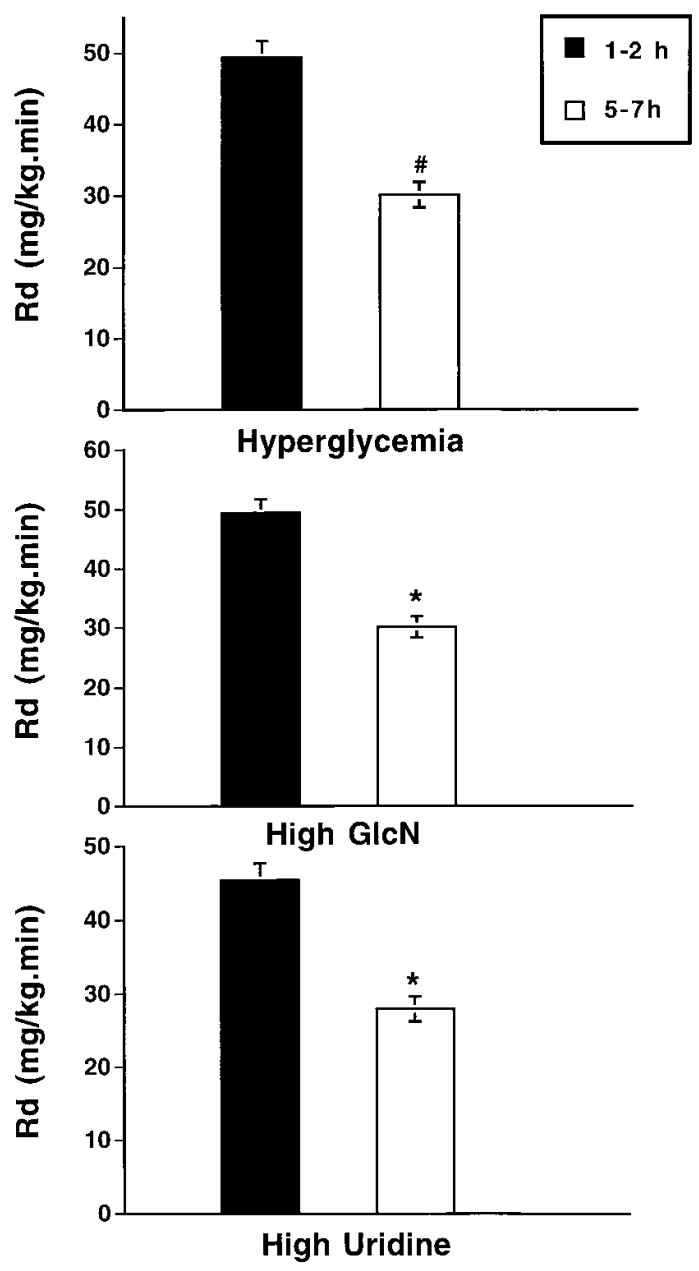

Figure 6. Peripheral glucose uptake $(R d)$ plotted for the initial and final 2-h intervals of euglycemic-hyperinsulinemic clamp studies either before and after 3-h hyperglycemia $(n=4)$ or during 7-h continuous infusions of glucosamine (High GlcN;n=10) or uridine (High Uridine; $n=4) .{ }^{*} P<0.05 ;{ }^{\sharp} P<0.01$ vs $0 \mathrm{~h}$.

In vivo metabolic parameters (Table III, Fig. 6). The plasma glucose concentrations were maintained at basal levels $(\sim 7$ $\mathrm{mM}$ ) during the euglycemic clamp studies and at $\sim 18 \mathrm{mM}$ during the hyperglycemic period of study 2 . Plasma FFA levels were markedly and similarly reduced by hyperinsulinemia in all groups. The infusion of GlcN ( $3 \mu \mathrm{mol} / \mathrm{kg}$ per min) over the 7-h studies increased the plasma GlcN concentration to $\sim 0.15$ $\mathrm{mM}$ in the GlcN group. The plasma insulin levels were maintained constant at $\sim 500 \mu \mathrm{U} / \mathrm{ml}$ throughout the $7 \mathrm{~h}$ in all groups.

Marked and similar decreases in insulin-mediated glucose disposal were induced by hyperglycemia (study 2), high GlcN (study 3 ) and high uridine (study 4). Fig. 6 depicts the marked decrease in the rates of peripheral glucose uptake (Rd) which occurred between the initial and the final $2 \mathrm{~h}$ of the studies in the three groups. The decreases in the rates of peripheral glucose uptake observed in all groups were again largely due to marked decreases in the rates of glycogen synthesis (hyperglycemia: $-50 \%$, GlcN: $-51 \%$ and uridine: $-56 \%$ ) while the rates of glycolysis were unaffected.
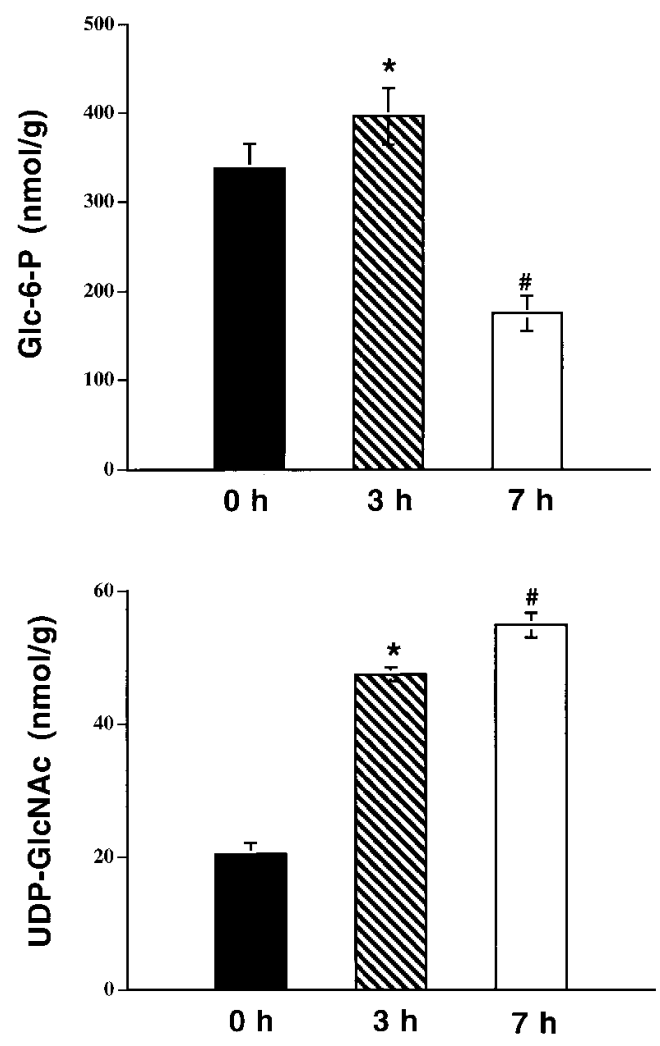

Figure 7. Skeletal muscle concentrations of Glc-6-P and UDPGlcNAc plotted for 7-h euglycemic-hyperinsulinemic clamp studies with saline infusion $(0 h ; n=6)$ or after $3 \mathrm{~h}(n=3)$ or $5 \mathrm{~h}(n=3)$ of hyperglycemia with basal insulin. ${ }^{*} P<0.01$; ${ }^{\#} P<0.001$ vs $0 \mathrm{~h}$.

Skeletal muscle metabolites. (Figs. 7 and 8, Table IV). Skeletal muscle glucose-6-phosphate concentrations were increased significantly above basal levels during the early hyperglycemic period of study 2 . However, there were marked reductions in skeletal muscle Glc-6P concentrations at the completion of the second insulin clamp studies $(7 \mathrm{~h})$ in all groups. A comparable almost twofold increase in the concentration of UDP-GlcNAc in skeletal muscle was demonstrated in all protocols at $7 \mathrm{~h}$, and during the early hyperglycemic period of study 2 (7 h: UDPGlcNAc $=54.8 \pm 1.9 \mathrm{nmol} /$ grams; $3 \mathrm{~h}: 47.4 \pm 1.0 \mathrm{nmol} / \mathrm{grams})$. The skeletal muscle levels of UDP-Glc and UDP-Gal were diminished by GlcN infusion and augmented with uridine infusion, but levels after hyperglycemia did not vary significantly from saline controls. Thus, the ratio of UDP-GlcNAc to UDPGlc concentrations in skeletal muscle was highest after GlcN infusion and lowest with uridine infusion.

\section{Discussion}

In the current studies, a prolonged elevation in the plasma FFA concentrations resulted in substantial and time-dependent impairments in insulin action on glucose uptake and glycogen synthesis. While these metabolic disturbances have been encountered previously with prolonged lipid infusion (7-10), the underlying biochemical mechanism(s) remains to be determined. In the attempt to advance our understanding of the pathophysiology of fat-induced insulin resistance, we exam- 

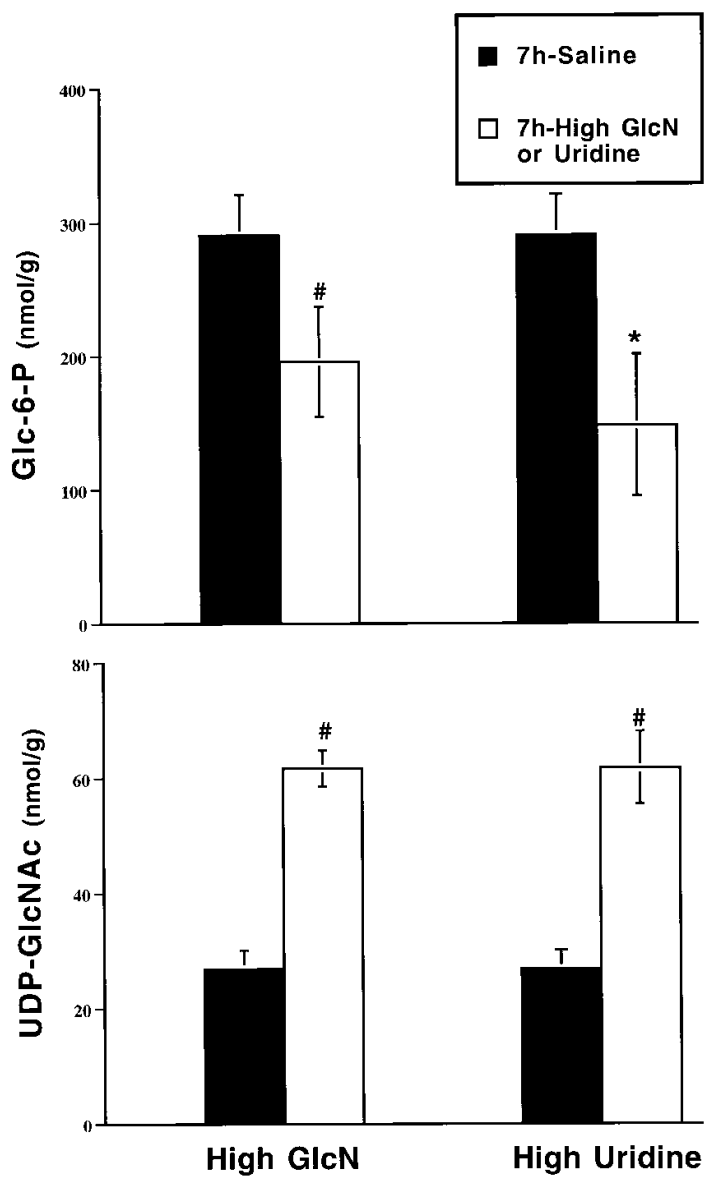

Figure 8. Skeletal muscle concentrations of Glc-6-P and UDPGlcNAc plotted after control euglycemic-hyperinsulinemic clamp studies with saline infusion (Saline; $n=6$ ) or after 7-h euglycemichyperinsulinemic clamp studies with continuous infusions of glucosamine (High GlcN; $n=10$ ) or uridine (High Uridine; $n=4)$. ${ }^{*} P<0.01 ;{ }^{\#} P<0.001$ vs $0 \mathrm{~h}$.

ined the temporal sequence of the resulting metabolic defects and their relationship to the hexosamine biosynthetic pathway. To this end, we assessed in vivo glucose fluxes and quantified the accumulation of key metabolites in skeletal muscle during prolonged lipid infusion. Experimental protocols were designed to answer the following four specific questions.

Are persistent elevations in circulating FFA levels required to maintain fat-induced insulin resistance? It is noteworthy that previous short-term studies of the effects of FFA on insulin action, while confirming the fat-induced inhibition of carbohydrate oxidation, were often unable to demonstrate a significant impairment in glucose uptake within $2 \mathrm{~h}$ of lipid infusion $(34,35)$. Conversely, studies in which there was prolonged exposure to elevated FFA levels for at least 3 or $4 \mathrm{~h}$ were associated with marked decreases in both glucose uptake and glycogen synthesis $(7-10,36)$. This implies that the multiple effects of hyperlipidemia on insulin action are time dependent $(7,8$, 37), and the impairments in insulin-mediated glucose uptake and glycogen synthesis probably require the prior development of an underlying metabolic alteration. If the mechanism for such decreases in glucose uptake and glycogen synthesis were inhibition of skeletal muscle hexokinase by fat-induced
Table IV. Skeletal Muscle UDP-Glc, UDP-Gal, $U D P-G l c N A c$, and UDP-GalNAc Concentrations after 7-h Hyperglycemic Clamp Studies with Low, Physiologic Insulin (Hyperglycemia), and 7-h Euglycemic-Hyperinsulinemic Clamp Studies with Infusions of Glucosamine (High GlcN) and Uridine (High Uridine)

\begin{tabular}{lrcc}
\hline & Hyperglycemia & High GlcN & High uridine \\
\hline UDP-Glc (nmol/gram) & $22.9 \pm 1.9$ & $12.7 \pm 1.2^{*}$ & $31.9 \pm 6.6^{*}$ \\
UDP-Gal (nmol/gram) & $8.9 \pm 0.4$ & $4.2 \pm 0.5^{*}$ & $12.7 \pm 2.0^{*}$ \\
UDP-GlcNAc (nmol/gram) & $54.8 \pm 1.9$ & $61.7 \pm 3.1$ & $58.7 \pm 6.3$ \\
UDP-GalNAc (nmol/gram) & $26.5 \pm 3.2$ & $13.3 \pm 0.6^{*}$ & $16.0 \pm 1.3^{*}$ \\
Ratio UDP-GlcNAc/UDP-Glc & $2.4 \pm 0.1$ & $5.2 \pm 0.6^{*}$ & $1.8 \pm 0.4^{*}$
\end{tabular}

$* P<0.01$ vs hyperglycemia. Values are mean \pm SE.

accumulation of Glc-6-P $(5,25,36)$, then sustained elevations in FFA levels would be required to maintain these deleterious effects on insulin action. The current study design was intended to specifically address this issue. Our hypothesis was that if the inhibition of carbohydrate oxidation due to increased availability of FFA were directly responsible for the decrease in insulin-mediated glucose uptake, lowering the plasma FFA concentrations below basal levels should normalize insulin action. As previously reported in humans $(7,8,10)$, the fat-induced reductions in glucose uptake and glycogen synthesis were significant by $\sim 3 \mathrm{~h}$ and maximal by $5 \mathrm{~h}$ of lipid infusion. However, they were both unaffected by the marked fall in circulating FFA levels in the subgroup in which lipid infusion was terminated at $5 \mathrm{~h}$. Thus, peripheral insulin resistance persisted for the remainder of the 7-h studies independent of the plasma FFA levels at the time of assessment. This observation is consistent with previous reports of a lag period for the onset of fat-induced impairment in insulin-mediated glucose uptake and suggests that the alterations induced by lipid infusion during the initial 3-5 h may lead to later and persistent defects in the action of insulin on skeletal muscle glucose uptake.

Are skeletal muscle Glc-6-P levels increased after the onset of fat-induced insulin resistance? After its phosphorylation to Glc-6-P, incoming glucose can be metabolized via two major pathways in skeletal muscle, glycolysis and glycogen synthesis. A decrease in the rate of glucose uptake may either be the direct consequence of a diminished rate of glucose transport/ phosphorylation or result from a reduction in the rate of utilization of Glc-6-P via glycogen synthesis and/or glycolysis. While the former mechanism would be expected to cause a drop in the steady-state concentration of Glc-6-P, the latter should be accompanied by a significant increase in the Glc-6-P levels. Indeed, the current studies revealed an early elevation followed by a marked decline in skeletal muscle levels of Glc-6-P with fat infusion, the latter coinciding with the onset of peripheral insulin resistance. This temporal pattern indicates that the deleterious effect of prolonged elevations in plasma FFA concentrations on glucose uptake and storage is due to a rate-determining defect at the level of glucose transport/phosphorylation. Kim et al. have recently reported that in vivo exposure to elevated free fatty acids resulted in early $(2 \mathrm{~h})$ elevations in skeletal muscle levels of Glc-6-P and decreased rates of glycolysis in conscious overnight fasted rats (36). However, the effect of lipid infusions of longer duration was not examined. In humans, 
using ${ }^{31} \mathrm{P}$-nuclear magnetic resonance spectroscopy, Roden et al. have shown an initial modest elevation followed by a marked and progressive decline in the skeletal muscle Glc-6-P levels during prolonged $(6 \mathrm{~h})$ lipid infusion (10). These authors concluded that a sustained elevation in plasma FFA concentration impairs insulin action on glucose uptake and glycogen synthesis via inhibition of glucose transport or phosphorylation (10). Previously, we emphasized the sequential acquisition of metabolic defects in carbohydrate oxidation, glycolysis, glucose uptake, and glycogen synthesis during 6-h lipid infusion in normal subjects (8). In the latter study, we were unable to demonstrate any increase in the skeletal muscle concentration of Glc-6-P after 2 and $4 \mathrm{~h}$ of lipid infusion, while a modest increase was demonstrated only after the onset of impaired glycogen synthase activity $(8,9)$. Consistent with the above evidence and in support of the rate-determining defect being at the level of glucose transport and/or phosphorylation, the decline in glucose uptake in the presence of elevated FFA levels in the present and in previous studies (8-10) is strongly correlated with the impaired ability of insulin to stimulate glycogen synthesis. Prolonged increases in GlcN availability resulted in decreased glucose transport and GLUT4 translocation both in isolated cell systems $(13,14,18)$ and in vivo $(15,16)$. Thus, future studies demonstrating that infusion of FFA resulted in a similar defect in GLUT4 translocation would further support a causal role for the GlcN pathway in FFA-induced insulin resistance.

Is there evidence that elevated FFA levels result in increased flux into the GlcN pathway (i.e., do skeletal muscle levels of UDP-GlcNAc rise during prolonged infusion of FFA)? Marshall et al. advanced the hypothesis that increased carbon flux (i.e., Fru-6-P) into the GlcN biosynthetic pathway would induce a defect in insulin action on glucose uptake $(13,14)$. Here we hypothesize that prolonged exposure to elevated FFA levels would promote increased flux of Fru-6-P into the glucosamine pathway, even in the presence of stable glucose flux into skeletal muscle. In fact, the early fat-induced inhibition of glucose oxidation and glycolysis is likely to transiently increase the size of the Fru-6-P pool and therefore its availability for the GFA enzyme (Fig. 1). Since we recently demonstrated that the accumulation of UDP- $N$-acetyl-hexosamines in skeletal muscle reflects the flux of carbons into the GlcN pathway and is highly correlated with the impairment in insulin action (22, 23 ), we used the accumulation of UDP-GlcNAc in skeletal muscle as an index of flux into this pathway during the lipid infusions. As noted, elevated FFA levels in the current studies were associated with early increases in the skeletal muscle concentrations of hexose-phosphates, and with an accumulation of UDP- $N$-acetyl-hexosamines which preceded and accompanied the defects in insulin-stimulated glucose uptake and glycogen synthesis. However, to hypothesize a cause-effect relationship between the fat-induced accumulation of end products of the GlcN pathway and impaired glucose uptake, it is necessary to demonstrate that increasing the concentration of UDP-GlcNAc in the absence of elevated FFA is sufficient to induce a similar degree of insulin resistance.

Could a comparable increase in skeletal muscle UDPGlcNAc achieved by different means also induce insulin resistance? While it is now well established that marked (greater than fourfold) elevations in the skeletal muscle concentration of UDP-GlcNAc induce peripheral insulin resistance $(15,22$, $23)$, it is not known whether the more moderate increases mea- sured in the present studies after lipid infusion could also impair insulin action on glucose uptake. To address this issue, we generated similar moderate increases in the levels of these endproducts using three alternate experimental conditions: hyperglycemia and increased availability of either GlcN or uridine. Hyperglycemia has been previously shown to moderately increase the skeletal muscle concentration of endproducts of the hexosamine pathway $(18,24)$. Our previous observation that increased GlcN availability induced peripheral insulin resistance in normal rats but had no additional inhibitory effects in diabetic rats implicated the glucosamine pathway as the potential mechanism by which hyperglycemia could induce resistance to insulin (15). In the current studies, the selected rate of GlcN infusion was 10 -fold lower than previously used to match the levels of UDP-GlcNAc encountered with hyperglycemia and increased FFA availability. Finally, an important relationship has been described between the intracellular uridine (UDP/UTP) pool and the hexosamine biosynthetic pathway, via its contribution to the uridylation of the $\mathrm{N}$-acetyl-hexosamines (23). We have recently demonstrated that prolonged infusion of uridine results in both increased accumulation of UDP-GlcNAc and peripheral insulin resistance, suggesting a stimulatory role for UDP/UTP in the formation of endproducts of the hexosamine pathway (23).

Indeed, all four experimental conditions had remarkably similar effects both on peripheral insulin action and on activation of the GlcN pathway. Increased availability of glucose (hyperglycemia), GlcN, and uridine resulted in marked and comparable resistance to the stimulatory effects of insulin on glucose uptake, over a parallel time course as was observed with hyperlipidemia. The design of the hyperglycemia protocol revealed that the onset of insulin resistance was again preceded by an increase in the skeletal muscle concentration of hexose-phosphates and of UDP- $N$-acetyl-hexosamines, while the formation of Glc-6-P in skeletal muscle decreased with prolonged exposure to all experimental conditions. Similar degrees of elevation of the endproducts of the hexosamine pathway in skeletal muscle indicated that carbon flux into this pathway was significantly and similarly increased by nearly twofold at the end of all study conditions. The similar time course of the effects of all conditions on skeletal muscle metabolites and on peripheral insulin action suggests that the observed suppression of insulin action by increased free fatty acid availability could be explained by increased flux through the GlcN pathway.

The evidence herein presented indicates that the glucosamine biosynthetic pathway may contribute to the pathophysiology of fat-induced insulin resistance. Indeed, this quantitatively small pathway of glucose utilization may link the rates of glucose and free fatty acid flux to the metabolic actions of insulin by functioning as a "fuel sensor" capable of decreasing the rate of glucose uptake when detecting increased availability of hexose-phosphates. However, while our observations provide experimental evidence in support of this hypothesis, further studies will be required to prove the causal relationship between the fat-induced increase in skeletal muscle UDPGlcNAc and the decrease in insulin-stimulated glucose uptake. In this regard, since the moderate (twofold) increase in muscle UDPGlcNAc observed in the present studies resulted in an impairment in glucose uptake which was similar to that previously reported with more marked increases in its concentration $(15,22)$, it will be particularly informative to determine 
whether the effects of prolonged FFA infusion, hyperglycemia, and GlcN on peripheral insulin resistance are additive. This also does not exclude the possibility that additional mechanisms might be responsible for the inhibitory effects of hyperlipidemia on peripheral insulin action. Skeletal muscle malonyl-CoA, whose levels are exquisitely sensitive to both insulin and glucose, appears to be an important fuel-sensing mechanism (38) which has recently been shown to be involved in the glucose-fatty acid cycle (39). The fatty acid composition of plasma membranes is another cellular factor that may influence the metabolic effects of insulin on skeletal muscle $(40,41)$.

In summary, prolonged increases in FFA availability result both in a marked impairment in the ability of insulin to promote skeletal muscle glucose transport and/or phosphorylation and in accumulation of endproducts of the hexosamine biosynthetic pathway. It is noteworthy that these moderate elevations in the skeletal muscle concentrations of UDP- $N$ acetyl-hexosamines are sufficient to induce marked peripheral insulin resistance even in the absence of sustained hyperlipidemia. Thus, we propose that a sustained increase in circulating FFA causes a specific defect in skeletal muscle glucose transport and/or phosphorylation via increased carbon flux into the GlcN biosynthetic pathway. This may provide a unifying hypothesis for both glucose- and fat-induced insulin resistance.

\section{Acknowledgments}

The authors wish to thank Robin Squeglia for excellent technical assistance.

This work was supported by grants from the National Institutes of Health (DK 45024 and DK48321), and the Juvenile Diabetes Foundation and by the Albert Einstein Diabetes Research and Training Center (DK 20541). Meredith Hawkins is the recipient of post-doctoral research fellowships from the Juvenile Diabetes Foundation and the Medical Research Council of Canada. Nir Barzilai is supported by a Clinical Investigator Award from the National Institutes of Health (KO8-AG00639). Luciano Rossetti is the recipient of a Career Scientist Award from the Irma T. Hirschl Trust.

\section{References}

1. DeFronzo, R.A., R.C. Bonadonna, and E. Ferrannini. 1992. Pathogenesis of NIDDM. A balanced overview. Diabetes Care. 15:318-368.

2. Reaven, G.M., C. Hollenbeck, C.-Y. Jeng, M.S. Wu, and Y.-D.I. Chen. 1988. Measurement of plasma glucose, free fatty acid, lactate, and insulin for $24 \mathrm{~h}$ in patients with NIDDM. Diabetes. 37:1020-1024.

3. Arslanian, S.A., and S.C. Kahlan. 1994. Correlations between fatty acid and glucose metabolism. Diabetes. 43:908-914.

4. Felber, J.P., E. Ferrannini, A. Golay, H.U. Meyer, D. Thiebaud, and R.A. Defronzo. 1987. Role of lipid oxidation in pathogenesis of insulin resistance of obesity and type II diabetes. Diabetes. 36:1341-1350.

5. Randle, P.J., P.B. Garland, C.N. Hales, and E.A. Newsholme. 1963. The glucose fatty acid cycle: Its role in insulin sensitivity and the metabolic disturbances of diabetes mellitus. Lancet. 1:785-789.

6. Randle, P.J., P.B. Garland, E.A. Newsholme, and C.N. Hales. 1965. The glucose fatty acid cycle in obesity and maturity onset diabetes mellitus. Ann. NY Acad. Sci. 131:324-333.

7. Boden, G., F. Jadali, J. White, Y. Liang, M. Mozzoli, X. Chen, E. Coleman, and C. Smith. 1991. Effects of fat on Insulin-stimulated carbohydrate metabolism in normal men. J. Clin. Invest. 88:960-966.

8. Boden, G., X. Chen, J. Ruiz, J.V. White, and L. Rossetti. 1994. Mechanisms of fatty acid-induced inhibition of glucose uptake. J. Clin. Invest. 93:24382446.

9. Kelley, D., M. Mokan, J.-A. Simoneau, and L. Mandarino. 1993. Interaction between glucose and free fatty acid metabolism in human skeletal muscle. J. Clin. Invest. 92:91-98.

10. Roden, M., T. Price, G. Perseghin, K. Petersen, D. Rothman, G. Cline, and G. Shulman. 1996. Mechanism of free fatty acid-induced insulin resistance in humans. J. Clin. Invest. 97:2859-2865.

11. Shulman, G., D. Rothman, T. Jue, P. Stein, R. DeFronzo, and R. Schulman. 1990. Quantitation of muscle glycogen synthesis in normal subjects and subjects with non-insulin dependent diabetes mellitus by ${ }^{13} \mathrm{C}$ nuclear magnetic resonance spectroscopy. N. Engl. J. Med. 322:223-228.

12. Rossetti, L., Y. Lee, J. Ruiz, S. Aldridge, H. Shamoon, and G. Boden 1993. Quantitation of glycolysis and skeletal muscle glycogen synthesis in humans. Am. J. Physiol. 265:E761-E769.

13. Marshall, S., V. Bacote, and R.R. Traxinger. 1991. Discovery of a metabolic pathway mediating desensitization of the glucose transport system: role of hexosamine biosynthesis in the induction of insulin resistance. J. Biol. Chem. 266:4706-4712.

14. Traxinger, R.R., and S. Marshall. 1991. Coordinate regulation of glutamine:fructose-6-P amidotransferase activity by insulin, glucose and glutamine. J. Biol. Chem. 266:10148-10154.

15. Rossetti, L., M. Hawkins, W. Chen, J. Gindi, and N. Barzilai. 1995. In vivo glucosamine infusion induces insulin resistance in normoglycemic but not in hyperglycemic conscious rats. J. Clin. Invest. 96:132-140.

16. Baron, A., J.-S. Zhu, J.-H. Zhu, H. Weldon, L. Maianu, and W.T. Garvey. 1995. Glucosamine induces insulin resistance in vivo by affecting GLUT4 translocation in skeletal muscle. J. Clin. Invest. 96:2792-2801.

17. Hebert, L., M. Daniels, J. Zhou, E. Crook, R. Turner, S. Simmons, J. Neidigh, J.-S. Zhu, A. Baron, and D. McClain. 1996. Overexpression of glutamine:fructose-6-phosphate amidotransferase in transgenic mice leads to insulin resistance. J. Clin. Invest. 98:930-936.

18. Robinson, K.A., D.A. Sens, and M.G. Buse. 1993. Pre-exposure to glucosamine induces insulin resistance of glucose transport and glycogen synthesis in isolated rat skeletal muscles. Diabetes. 42:1333-1346.

19. Giaccari, A., L. Morviducci, D. Zorretta, P. Sbraccia, F. Leonetti, S. Caiola, A. Buongiorno, and R.C. Bonadonna. 1995. In vivo effects of glucosamine on insulin secretion and insulin sensitivity in the rat: possible relevance to the maladaptive responses to chronic hyperglycaemia. Diabetologia. 38:518-524.

20. Crook, E.D., M.C. Daniels, T.M. Smith, and D.A. McClain. 1993. Regulation of insulin-stimulated glycogen synthase activity by overexpression of Glutamine:fructose-6-phosphate amidotransferase in rat-1 fibroblasts. Diabetes. 42:1289-1296.

21. Rossetti, L., D. Smith, G. Schulman, D. Papachristou, and R. DeFronzo. 1987. Correction of hyperglycemia with phlorizin normalizes tissue sensitivity to insulin in diabetic rats. J. Clin. Invest. 79:1510-1515.

22. Hawkins, M., N. Barzilai, W. Chen, I. Angelov, M. Hu, P. Cohen, and L. Rossetti. 1996. Increased Hexosamine availability similarly impairs the action of insulin and IGF-1 on glucose disposal. Diabetes. 45:1734-1743.

23. Hawkins, M., I. Angelov, R. Liu, N. Barzilai, and L. Rossetti. 1997. The tissue concentration of UDP- $N$-acetyl-glucosamine modulates the stimulatory effect of insulin on skeletal muscle glucose uptake. J. Biol. Chem. 272:4889-4895.

24. Buse, M.G., K.A. Robinson, B.A. Marshall, and M.M. Mueckler. 1996. Differential effects of GLUT1 or GLUT4 overexpression on hexosamine biosynthesis by muscles of transgenic mice. J. Biol. Chem. 271:23197-23202.

25. Randle, P.J., A.L. Kerbey, and J. Espinal. 1988. Mechanisms decreasing glucose oxidation in diabetes and starvation: role of lipid fuels and hormones. Diabetes \& Metab. Rev. 4:623-638.

26. Liu, Y., and K. Uyeda. 1996. A mechanism for fatty acid inhibition of glucose utilization in liver. Role of xylulose-5-P. J. Biol. Chem. 271:8824-8830.

27. Rossetti, L., and M.R. Laughlin. 1989. Correction of chronic hyperglycemia with vanadate, but not phlorizin, normalizes in vivo glycogen repletion and in vitro glycogen synthase activity in diabetic skeletal muscle. J. Clin. Invest. 84: 892-899.

28. Rossetti, L., and A. Giaccari. 1990. Relative contribution of glycogen synthesis and glycolysis to insulin-mediated glucose uptake. A dose-response euglycemic clamp study in normal and diabetic rats. J. Clin. Invest. 85:17851792.

29. Rossetti, L., and M. Hu. 1993. Skeletal muscle glycogenolysis is more sensitive to insulin than is glucose transport/phosphorylation. Relation to the insulin-induced inhibition of hepatic glucose production. J. Clin. Invest. 92: 2963-2974.

30. Michal, G. 1985. D-glucose-6-phosphate. In Methods of Enzymatic Analysis. Vol. VI. N.U. Bergmeyer, editor. VCH Publishers, Weinheim, Germany. 191-198.

31. Giaccari, A., and L. Rossetti. 1989. Isocratic high-performance liquid chromatographic determination of the concentration and specific activity of phosphoenolpyruvate and uridine diphosphate glucose in tissue extracts. J. Chromatogr. 497:69-78.

32. Giaccari, A., and L. Rossetti. 1992. Predominant role of gluconeogenesis in the hepatic glycogen repletion of diabetic rats. J. Clin. Invest. 89:36-45.

33. Anumula, K.R., and P.B. Taylor. 1991. Quantitative determination of phenyl isothiocyanate-derivatized amino sugars and amino sugar alcohols by high-performance liquid chromatography. Anal. Biochem. 197:113-120.

34. Bevilacqua, S., R. Bonadonna, G. Buzzigoli, C. Boni, D. Ciociaro, F. Maccari, M. Giorico, and E. Ferrannini. 1987. Acute elevation of free fatty acid levels leads to hepatic insulin resistance in obese subjects. Metab. Clin. Exp. 36: 502-506. 
35. Wolfe, B., S. Klein, E. Peters, B. Schmidt, and R. Wolfe. 1988. Effect of elevated free fatty acids on glucose oxidation in normal humans. Metab. Clin. Exp. 37:323-329.

36. Kim, J., J. Wi, and J. Youn. 1996. Plasma free fatty acids decrease insulin-stimulated skeletal muscle glucose uptake by suppressing glycolysis in conscious rats. Diabetes. 45:446-453.

37. Bonadonna, R., K. Zych, C. Boni, E. Ferrannini, and R. DeFronzo. 1989. Time dependence of the interaction between lipid and glucose in humans. Am. J. Physiol. 257:E49-E56.

38. Saha, A., T. Kurowski, and N. Ruderman. 1995. A malonyl-CoA fuelsensing mechanism in muscle: effects of insulin, glucose and denervation. Am.
J. Physiol. 269:E283-E289.

39. Saha, A., D. Vavvas, T. Kurowski, A. Apazidis, L. Witters, E. Shafrir and N. Ruderman. 1996. The malonyl-CoA fuel-sensing and signaling mechanism in skeletal muscle: its link to cell citrate and the glucose fatty acid cycle. Diabetes. 45(Suppl. 2):12a.

40. Ginsberg, B., J. Jabour, and A. Spector. 1982. Effect of alterations in membrane lipid unsaturation on the properties of the insulin receptor of Ehrlich ascites fluid cells. Biochem. Biophys. Acta. 690:157-164.

41. Borkman, M., L. Storlien, D. Pan, A. Jenkins, D. Chisholm, and L. Campbell. 1993. The relation between insulin sensitivity and the fatty-acid composition of skeletal muscle phospholipids. N. Engl. J. Med. 328:238-244. 